SLAC-PUB-10156

hep-th/0309044

\title{
On effective superpotentials and Kutasov duality
}

\author{
Matthias Klein*, Sang-Jin $\operatorname{Sin}^{\dagger}$ \\ *† Theory Group, SLAC, Stanford University, Stanford, CA 94309, USA. \\ † Department of Physics, Hanyang University, Seoul, 133-791, Korea.
}

\begin{abstract}
We derive the effective superpotential for an $\mathcal{N}=1 S U\left(N_{c}\right)$ gauge theory with one massless adjoint field and $N_{f}$ massless fundamental flavors and cubic tree-level superpotential for the adjoint field. This is a generalization of the Affleck-Dine-Seiberg superpotential to gauge theories with one massless adjoint matter field. Using Kutasov's generalization of Seiberg duality, we then find the effective superpotential for a related theory with massive fundamental flavors.
\end{abstract}

September 2003

\footnotetext{
*E-mail: mklein@slac.stanford.edu

${ }^{\dagger}$ E-mail: sjsin@hanyang.ac.kr
} 


\section{Introduction}

Nonperturbative superpotentials are important because they lead to an understanding of interesting quantum effects in supersymmetric field theories and because of their phenomenological implications for supersymmetric models. Furthermore, they are an ideal arena to test our computational methods in supersymmetric field theories. We will use a combination of several nonperturbative methods to determine the effective superpotential for a class of supersymmetric gauge theories with matter in the adjoint and fundamental representations. In earlier days, Affleck, Dine and Seiberg [1] were able to determine the effective nonperturbative superpotential for SQCD with $N_{f}<N_{c}$, this is an $\mathcal{N}=1 S U\left(N_{c}\right)$ gauge theory with $N_{f}$ quark flavors, by performing an explicit instanton calculation.

During the last decade many powerful techniques have been developed to compute effective superpotentials for broad classes of supersymmetric field theories. Seiberg showed [2] that in many cases the nonperturbative superpotential is completely determined by the symmetries and some physical boundary conditions. He also found [3] that the 't Hooft anomaly matching conditions [4] are an important constraint when determining the low-energy spectrum of gauge invariant operators and used this to establish an electric-magnetic duality [5] relating two different $\mathcal{N}=1$ gauge theories in the far IR. Some of these results were subsequently generalized to supersymmetric gauge theories with different gauge groups and/or matter content. Of particular interest is a model first discussed by Kutasov [6] containing a massless adjoint field and massless fundamental fields. However, although many nonperturbative results for the Kutasov model have been obtained, including a dual description in terms of magnetic variables [6, 7, 8, an effective superpotential generalizing the result of Affleck, Dine and Seiberg for SQCD [1] is still missing. ${ }^{1}$

Sometime ago, Csáki and Murayama showed that, for a special choice of the numbers of colors and flavors, an effective superpotential for the confined degrees of freedom of the Kutasov model can be obtained by analyzing the classical constraints for the gauge invariant operators [12. It is interesting that under certain conditions the full nonperturbative superpotential is determined by the requirement to reproduce the classical constraints via its equations of motion.

In this article, we use the method developed by Csáki and Murayama [12] to determine the low-energy effective nonperturbative superpotential for the model discussed by Kutasov [6]. Our result is a generalization of the effective superpotential discovered by Affleck, Dine and Seiberg to supersymmetric gauge theories containing matter fields in the adjoint and

\footnotetext{
${ }^{1}$ Much more is known for gauge theories containing massive adjoint and fundamental fields. See, e.g., [9, 11, 10] and references therein.
} 
fundamental representations. We use the results of [12] for an $\mathcal{N}=1 S U\left(N_{c}\right)$ gauge theory with $N_{f}$ massless fundamental flavors and one massless adjoint field $\Phi$ with $W_{\text {tree }}=h \operatorname{tr} \Phi^{3}$. For $2 N_{f}=N_{c}+1$, the theory confines without chiral symmetry breaking. The effective superpotential in terms of confined degrees of freedom was determined in [12. Starting from this result, we obtain the effective superpotential for $2 N_{f}<N_{c}$ :

$$
W_{\text {eff }}=\left(\frac{h^{N_{c}} \Lambda^{2\left(2 N_{c}-N_{f}\right)}}{\left(\operatorname{det} M_{2}\right)^{2}}\right)^{\frac{1}{N_{c}-2 N_{f}}} \operatorname{Tr}\left(M_{1} M_{2}{ }^{-1}\right),
$$

where $M_{1}, M_{2}$ are the gauge invariant meson operators. This generalizes the Affleck-DineSeiberg superpotential for SQCD. The fact that the effective superpotential (1.1) contains only one term, in an expansion in powers of $\Lambda^{\left(2 N_{c}-N_{f}\right) /\left(N_{c}-2 N_{f}\right)}$, is a consequence of the global symmetries and physical boundary conditions in the weak and strong coupling limits. The situation is more complicated if the tree-level superpotential is of the form $W_{\text {tree }}=h \operatorname{tr} \Phi^{k+1}$ for $k>2$. In these cases the effective superpotential contains several terms. Interestingly, the additional terms do not modify the classical constraints in the confining phase.

Using the electric-magnetic duality of the Kutasov model [6, 7, 8, one can find the (leading term of) the effective superpotential of a related theory which has the same gauge group and matter content but a different tree-level superpotential, which gives mass to the fundamental flavors and couples them to the adjoint field. Thus, one obtains nonperturbative information about an $\mathcal{N}=1$ gauge theory with massless adjoint matter and massive fundamental fields. The idea is that the effective superpotential for the electric theory can be translated into a corresponding effective superpotential of the dual magnetic theory. On the other hand, the magnetic theory can be viewed as the electric theory of a different model with a different tree-level superpotential.

In section 2 , we briefly review how the form of effective superpotentials can be determined by symmetry considerations. We derive the dependence on the masses and coupling parameters of the effective superpotential of an $\mathcal{N}=1 S U\left(N_{c}\right)$ gauge theory, with massive fundamental fields and a massive or massless adjoint field. In section 3, we discuss the case where both, the adjoint and fundamental fields, are massless. Building upon results of Csáki and Murayama [12], we are able to determine the exact low-energy effective superpotential if the tree-level superpotential for the adjoint field is cubic. In section 4, we apply electricmagnetic Kutasov duality to the theory with massless adjoint and fundamental fields to obtain the leading term of the effective superpotential of the theory with massless adjoint and massive fundamental fields. In an appendix, we explain how to generalize some of the results of section 3 and 4 to the case with tree-level superpotential $W_{\text {tree }}=h \operatorname{tr} \Phi^{k+1}$ for $k>2$. We also determine the constraints on the quantum moduli space and show that they coincide with the classical constraints. 


\section{Effective superpotentials from symmetries}

We start by using symmetries to constrain the form of the effective superpotential for an $\mathcal{N}=1$ supersymmetric $S U\left(N_{c}\right)$ gauge theory with one chiral superfield $\Phi$ in the adjoint representation and $N_{f}$ quark flavors - i.e., $N_{f}$ chiral superfields $Q$ in the fundamental representation and $N_{f}$ chiral superfields $\bar{Q}$ in the antifundamental representation - and tree-level superpotential

$$
W_{\text {tree }}=\sum_{l=1}^{k} \operatorname{Tr}\left(m_{l} \bar{Q} \Phi^{l-1} Q\right)+\frac{1}{2} m_{\Phi} \operatorname{tr} \Phi^{2}+\frac{1}{k+1} h \operatorname{tr} \Phi^{k+1},
$$

where $k$ is some integer $<N_{c}, h$ is a coupling parameter of mass dimension $(2-k)$ and the $m_{l}$ are matrix valued coupling parameters of mass dimension $(2-l)$. We denoted the flavor trace by ' $T r$ ' to distinguish it from the color trace denoted by 'tr'. This model is a deformation of the model analyzed by Kutasov and Schwimmer [7] and has previously been discussed in [8. Although many of our results are valid for general $k$, we will mostly be interested in the $k=2$ case, which corresponds to a cubic superpotential. The form of the effective superpotential can be determined using a method introduced by Seiberg [2], where one treats the parameters as background fields that carry charges under the global symmetries of the theory without superpotential. If the tree-level superpotential vanishes, the theory has a large global symmetry. The full global symmetry can be preserved by all interactions in (2.1) if appropriate charges are assigned to the parameters. As a consequence, any effective superpotential which is a function of the parameters and gauge invariant combinations of the massless fields has to be invariant under the full global symmetry. Some of the classical symmetries are anomalous at the quantum level. But the effective superpotential can be rendered invariant even under these symmetries if one assigns appropriate charges to the dynamically generated scale $\Lambda .^{2}$

In our case, the theory with $W_{\text {tree }}=0$ has a global symmetry

$$
G=S U\left(N_{f}\right)_{L} \times S U\left(N_{f}\right)_{R} \times U(1)_{R} \times U(1)_{\Phi} \times U(1)_{A} \times U(1)_{B} .
$$

Under this symmetry, the fields transform as shown in table 1 We chose some convenient values for the Abelian charges of $\Phi$ and $Q$. The remaining charges are determined by the anomaly freedom of $U(1)_{R}$ and $U(1)_{B}$. For general R-charge $R_{\Phi}$ of the adjoint field, one finds that the $U(1)_{R}$ symmetry is anomaly free if $Q, \bar{Q}$ have charge $R_{Q}=1-R_{\Phi} \frac{N_{c}}{N_{f}}$. The baryon symmetry $U(1)_{B}$ is anomaly free if $Q$ and $\bar{Q}$ have opposite charges. The anomalies of $U(1)_{\Phi}$ and $U(1)_{A}$ can be cured by assigning charges $2 N_{c}$ and $2 N_{f}$ to $\Lambda^{b}$, where $b=2 N_{c}-N_{f}$ is the one-loop coefficient of the beta-function. The charges of the parameters of the non-vanishing superpotential (2.1) are then easily determined. The results are summarized in table 1 .

\footnotetext{
${ }^{2}$ For a pedagogical review of these techniques see [13].
} 


\begin{tabular}{c|cccccc} 
& $S U\left(N_{f}\right)_{L}$ & $S U\left(N_{f}\right)_{R}$ & $U(1)_{R}$ & $U(1)_{\Phi}$ & $U(1)_{A}$ & $U(1)_{B}$ \\
\hline$Q$ & $\square$ & $\mathbf{1}$ & $1-\frac{N_{c}}{N_{f}}$ & 0 & 1 & 1 \\
$\bar{Q}$ & $\mathbf{1}$ & $\square$ & $1-\frac{N_{c}}{N_{f}}$ & 0 & 1 & -1 \\
$\Phi$ & $\mathbf{1}$ & $\mathbf{1}$ & 1 & 1 & 0 & 0 \\
$m_{\Phi}$ & $\mathbf{1}$ & $\mathbf{1}$ & 0 & -2 & 0 & 0 \\
$h$ & $\mathbf{1}$ & $\mathbf{1}$ & $-(k-1)$ & $-(k+1)$ & 0 & 0 \\
$m_{l}$ & $\mathbf{\square}$ & $\square$ & $\frac{2 N_{c}}{N_{f}}-(l-1)$ & $-(l-1)$ & -2 & 0 \\
$\Lambda^{b}$ & $\mathbf{1}$ & $\mathbf{1}$ & 0 & $2 N_{c}$ & $2 N_{f}$ & 0
\end{tabular}

Table 1: Charges of fields and parameters in (2.1) under the global symmetries.

The effective superpotential obtained after integrating out the massive fields $\Phi, Q$ and $\bar{Q}$ only depends on the parameters $m_{\Phi}, h, m_{l}, \Lambda$, and can be written as

$$
W_{\text {eff }} \sim \Lambda^{b x / N_{c}} \prod_{l=1}^{k} m_{l}^{y_{l}} m_{\Phi}^{w} h^{z},
$$

for some numbers $x, y_{l}, w, z$, where we suppressed the flavor indices on $m_{l}$ which should be contracted appropriately. Invariance under $U(1)_{R} \times U(1)_{\Phi} \times U(1)_{A}$ implies the following conditions:

$$
\sum_{l=1}^{k} y_{l}=x \frac{N_{f}}{N_{c}}, \quad \sum_{l=1}^{k} y_{l}(l-1)=2 x-2 w-z(k+1), \quad z+w=1 .
$$

This determines three of the $k+3$ exponents in (2.3).

To be more specific, let us now consider the case $k=2$ in more detail. One finds that the effective superpotential has to be of the form

$$
W_{\text {eff }}=m_{\Phi} \sum_{x, z} c_{x, z}\left(\Lambda^{b} \operatorname{det} m_{1}\right)^{\frac{x}{N_{c}}}\left[\operatorname{Tr}\left(\left(m_{2} m_{1}^{-1}\right)^{2(x-1)-z}\right)+\text { multi-trace }\right]\left(\frac{h}{m_{\Phi}}\right)^{z}
$$

to be invariant under the global symmetries. Multi-trace contributions like $\left(\operatorname{Tr}\left(m_{2} m_{1}^{-1}\right)\right)^{n}$ are allowed by the symmetries. From the matrix model approach to determining the effective superpotential, one knows [14] that only diagrams with at most one boundary ${ }^{3}$ contribute to the Veneziano-Yankielowicz-Dijkgraaf-Vafa superpotential $W(S)$ [15, 16] containing the

\footnotetext{
${ }^{3}$ In the 't Hooft double-line formalism a boundary corresponds to a trace over fields in the fundamental representation.
} 
glueball superfield $S$. Thus each term in $W(S)$ can have at most one trace over flavor indices. However, integrating out $S$ from $W(S)$ yields various multi-trace contributions in $W_{\text {eff }}$. The coefficients $c_{x, z}$ are undetermined by the symmetries. The dynamical scale $\Lambda_{L}$ of the low-energy theory is related to the high-energy scale $\Lambda$ by

$$
\Lambda_{L}^{3 N_{c}}=\Lambda^{2 N_{c}-N_{f}} m_{\Phi}^{N_{c}} \operatorname{det} m_{1}
$$

Inserting this relation into (2.5), we find

$$
W_{\mathrm{eff}}=\Lambda_{L}^{3} \sum_{x, z} c_{x, z}\left[\operatorname{Tr}\left(\left(\alpha \Lambda_{L}^{3}\right)^{x-1} \beta^{z}\right)+\text { multi-trace }\right]
$$

with $\alpha=m_{2} m_{1}^{-1} m_{2} m_{1}^{-1} / m_{\Phi}$ and $\beta=m_{1} m_{2}^{-1} h / m_{\Phi}$. The coefficients in this expansion can be determined either by performing a matrix model computation similar to one discussed in [17] or by solving the factorization equations of the corresponding Seiberg-Witten curve [18, 19, 11. This will be done in a separate publication [20].

If the adjoint field $\Phi$ is massless, we have have to set $w=0$ in (2.3), which yields $z=1$ in (2.4). The low-energy scale $\Lambda_{L}$ is now given by

$$
\Lambda_{L}^{2 N_{c}}=\Lambda^{2 N_{c}-N_{f}} \operatorname{det} m_{1}
$$

Inserting this and the condition $z=1$ into (2.5), we find

$$
W_{\text {eff }}=h \Lambda_{L}^{3} \sum_{x} c_{x}\left[\operatorname{Tr}\left(\left(\Lambda_{L} m_{2} m_{1}^{-1}\right)^{2 x-3}\right)+\text { multi-trace }\right] .
$$

Note that the effective superpotential has to be linear in $h$ to be consistent with the global symmetries. ${ }^{4}$ The coefficients $c_{x}$ cannot be determined by symmetry considerations. However, using the electric-magnetic duality discovered by Kutasov [6], one can show that the leading term in the expansion (2.9) is $\Lambda^{4}$; the coefficient $c_{1}$ vanishes. This will be discussed in section 4 .

\section{Effective superpotentials from classical constraints}

We would now like to find the effective superpotential for the model first discussed by Kutasov and Schwimmer [6, 7]. This is an $\mathcal{N}=1$ supersymmetric $S U\left(N_{c}\right)$ gauge theory with one adjoint field $\Phi, N_{f}$ quark flavors $Q, \bar{Q}$ and tree-level superpotential

$$
W_{\text {tree }}=\frac{1}{k+1} h \operatorname{tr} \Phi^{k+1},
$$

\footnotetext{
${ }^{4}$ This is a manifestation of the linearity principle discussed in [21].
} 


\begin{tabular}{c|cccccc} 
& $S U\left(N_{f}\right)_{L}$ & $S U\left(N_{f}\right)_{R}$ & $U(1)_{R}$ & $U(1)_{\Phi}$ & $U(1)_{A}$ & $U(1)_{B}$ \\
\hline$Q$ & $\square$ & $\mathbf{1}$ & $1-\frac{2 N_{c}}{(k+1) N_{f}}$ & 0 & 1 & $\frac{1}{N_{c}}$ \\
$\bar{Q}$ & $\mathbf{1}$ & $\square$ & $1-\frac{2 N_{c}}{(k+1) N_{f}}$ & 0 & 1 & $-\frac{1}{N_{c}}$ \\
$\Phi$ & $\mathbf{1}$ & $\mathbf{1}$ & $\frac{2}{k+1}$ & 1 & 0 & 0 \\
$u_{n}$ & $\mathbf{1}$ & $\mathbf{1}$ & $\frac{2 n}{k+1}$ & $n$ & 0 & 0 \\
$h$ & $\mathbf{1}$ & $\mathbf{1}$ & 0 & $-(k+1)$ & 0 & 0 \\
$M_{l}$ & $\square$ & $\mathbf{0}$ & $\left(-\frac{2 N_{c}}{N_{f}}+k+l\right) \frac{2}{k+1}$ & $l-1$ & 2 & 0 \\
$\Lambda^{b}$ & $\mathbf{1}$ & $\mathbf{1}$ & 0 & $2 N_{c}$ & $2 N_{f}$ & 0
\end{tabular}

Table 2: Charges of elementary and composite fields of the Kutasov model (3.1). For convenience, we have chosen the R-charge of $\Phi$ to be such that $h$ is neutral under $U(1)_{R}$.

where $k$ is some integer $<N_{c}$ and $h$ is a parameter of mass dimension $2-k$. The gauge invariant operators that generate the chiral ring are [7, 8]

$$
M_{l} \equiv \bar{Q} \Phi^{l-1} Q, \quad l=1, \ldots, k, \quad u_{n} \equiv \frac{1}{n} \operatorname{tr} \Phi^{n}, \quad n=2, \ldots, k .
$$

The charges of the elementary and composite fields are shown in table 2. If $k N_{f} \geq N_{c}$, then there are also baryonic operators. To define them, we first introduce dressed quark operators:

$$
Q_{l} \equiv \Phi^{l-1} Q, \quad \bar{Q}_{l} \equiv \bar{Q} \Phi^{l-1} .
$$

The baryons carry $k N_{f}-N_{c}$ flavor indices and are given by

$$
\begin{aligned}
& B^{\left(n_{1}, \ldots, n_{k}\right)} \equiv\left(Q_{1}\right)^{n_{1}}\left(Q_{2}\right)^{n_{2}} \cdots\left(Q_{k}\right)^{n_{k}}, \quad \bar{B}^{\left(\bar{n}_{1}, \ldots, \bar{n}_{k}\right)} \equiv\left(\bar{Q}_{1}\right)^{\bar{n}_{1}}\left(\bar{Q}_{2}\right)^{\bar{n}_{2}} \cdots\left(\bar{Q}_{k}\right)^{\bar{n}_{k}} \\
& \text { with } \sum_{l=1}^{k} n_{k}=\sum_{l=1}^{k} \bar{n}_{k}=N_{c} .
\end{aligned}
$$

We suppressed all color and flavor indices. The integers $\left\{n_{l}\right\}$ label the different types of baryons. Each of them carries $k N_{f}-N_{c}$ flavor indices which we have not displayed. The exponents denote the powers to which the dressed quarks appear. The color indices are contracted with a rank $N_{c}$ epsilon-tensor. The $N_{c}$ flavor indices are contracted with $k$ rank $N_{f}$ epsilon-tensors, leaving $k N_{f}-N_{c}$ free indices. (The first rank $N_{f}$ epsilon-tensor is contracted with $\left(Q_{1}\right)^{n_{1}}$, the second with $\left(Q_{2}\right)^{n_{2}}$, etc.)

Murayama and Csáki observed [12] that for $k N_{f}=N_{c}+1$, the low-energy effective theory of this model is described by confined degrees of freedom and that the point of unbroken 
chiral symmetry is not removed from the quantum moduli space. This is a generalization of a similar result for SQCD with $N_{f}=N_{c}+1$ by Seiberg [3]. The confined degrees of freedom in the latter case obey the constraints

$$
M_{\bar{\imath}}{ }^{j} B_{j}=0, \quad \bar{B}^{\bar{\imath}} M_{\bar{\imath}}{ }^{j}=0, \quad(\operatorname{cof} M)_{j}{ }^{\overline{ }}=B_{j} B^{\bar{\imath}},
$$

where $i, j$ are $S U\left(N_{f}\right)_{L}$ indices, $\bar{\imath}, \bar{\jmath}$ are $S U\left(N_{f}\right)_{R}$ indices and the cofactor is defined by cof $M=(\partial / \partial M) \operatorname{det} M$. The authors of [12] developed a method how to determine the analogous classical constraints in the Kutasov model explicitly. The trick is to realize that all the constraints involving quark fields can be obtained by treating the dressed quarks (3.3) as the only independent degrees of freedom. By assembling the dressed quarks $Q_{l}$ in one vector of an enlarged flavor space

$$
\mathcal{Q}=\left(Q_{1}, \ldots, Q_{k}\right), \quad \overline{\mathcal{Q}}=\left(\bar{Q}_{1}, \ldots, \bar{Q}_{k}\right)
$$

and at the same time forgetting about the $\Phi$ degrees of freedom, one has effectively mapped the Kutasov model to SQCD with $k N_{f}$ flavors. Similarly, the $k N_{f}$ baryons ${ }^{5}$ (3.4) can be assembled in one vector of the enlarged flavor space:

$$
\mathcal{B}=\left(B_{1}, \ldots, B_{k}\right), \quad \overline{\mathcal{B}}=\left(\bar{B}_{1}, \ldots, \bar{B}_{k}\right),
$$

where $B_{1} \equiv B^{\left(N_{f}-1, N_{f}, \ldots, N_{f}\right)}, B_{2} \equiv B^{\left(N_{f}, N_{f}-1, \ldots, N_{f}\right)}, \ldots, B_{k} \equiv B^{\left(N_{f}, \ldots, N_{f}, N_{f}-1\right)}$. Finally, one forms the enlarged meson matrix

$$
\mathcal{M}=\overline{\mathcal{Q}} \mathcal{Q}
$$

Now, the constraints for the Kutasov model with $k N_{f}=N_{c}+1$ follow directly from (3.5) and read

$$
\mathcal{M}_{\bar{I}}^{J} \mathcal{B}_{J}=0, \quad \overline{\mathcal{B}}^{\bar{I}} \mathcal{M}_{\bar{I}}^{J}=0, \quad(\operatorname{cof} \mathcal{M})_{J}^{\bar{I}}=B_{J} B^{\bar{I}}
$$

where the capital indices run from 1 to $k N_{f}$. There are some additional constraints following from the $\Phi$ equation of motion, which cannot be obtained in this picture of SQCD with $k N_{f}$ flavors. Using $\partial W_{\text {tree }} / \partial \Phi=0$, one can show [12] that only $B_{k}$ and $\bar{B}_{k}$ are non-zero in (3.7) and that $\bar{Q}_{l} Q_{l^{\prime}}=0$ if $l+l^{\prime}>k+1$. Thus, $\mathcal{M}, \mathcal{B}, \overline{\mathcal{B}}$ are of the form

$$
\mathcal{M}=\left(\begin{array}{ccccc}
M_{1} & M_{2} & \ldots & M_{k-1} & M_{k} \\
M_{2} & \ddots & M_{k-1} & M_{k} & \\
\vdots & M_{k-1} & M_{k} & & \\
M_{k-1} & M_{k} & & 0 & \\
M_{k} & & & &
\end{array}\right)
$$

\footnotetext{
${ }^{5}$ In the case $k N_{f}=N_{c}+1$, there are $k$ different choices for the $\left(n_{1}, \ldots, n_{k}\right)$ and each baryon carries one $S U\left(N_{f}\right)$ index.
} 


$$
\mathcal{B}=\left(0, \ldots, 0, B_{k}\right), \quad \overline{\mathcal{B}}=\left(0, \ldots, 0, \bar{B}_{k}\right)
$$

In the case of SQCD with $N_{f}=N_{c}+1$ flavors, it is easy to see [3] that the equations of motion of the confining superpotential

$$
W_{\mathrm{conf}}=\frac{\bar{B} M B-\operatorname{det} M}{\Lambda^{3 N_{c}-N_{f}}}
$$

are just the classical constraints (3.5). It has been shown that the classical constraints do not receive any quantum corrections and that (3.11) is the exact effective superpotential at low energies.

Let us determine the effective superpotential for the confined degrees of freedom of the Kutasov model [12. Comparing the classical constraints (3.9) to those of SQCD, one would guess that the former are reproduced by a superpotential of the form

$$
W_{\text {conf }} \sim \overline{\mathcal{B}} \mathcal{M B}-\operatorname{det} \mathcal{M}
$$

However, there are two problems with this approach. First, this superpotential does not have the correct R-charge. From the charges summarized in table 2 and the definitions of $\mathcal{M}, \mathcal{B}$, $\overline{\mathcal{B}}$, one finds that $\overline{\mathcal{B}} \mathcal{M B}$ has R-charge $2+\frac{2(k-1)}{k+1} .{ }^{6}$ Second, one cannot derive a superpotential for the physical degrees of freedom $M_{l}, B_{k}, \bar{B}_{k}$ from (3.12), since inserting the constraints (3.10) into (3.12) gives $W_{\text {conf }} \sim\left(\operatorname{det} M_{k}\right)^{k}$. The constraints (3.9) can only be obtained from (3.12) by first deriving the equations of motion and then inserting (3.10).

These difficulties can be overcome by introducing ad-hoc a new meson matrix

$$
\widehat{\mathcal{M}}=\left(\begin{array}{ccccc} 
& & & & M_{1} \\
& & & M_{1} & M_{2} \\
& & M_{1} & M_{2} & \vdots \\
& M_{1} & M_{2} & \ddots & M_{k-1} \\
M_{1} & M_{2} & \cdots & M_{k-1} & M_{k}
\end{array}\right) .
$$

A superpotential of the form

$$
W_{\text {conf }} \sim \overline{\mathcal{B}} \widehat{\mathcal{M}} \mathcal{B}-\widehat{\mathcal{M}} \operatorname{cof} \mathcal{M}
$$

\footnotetext{
${ }^{6}$ One might think that it is possible for $\overline{\mathcal{B}} \mathcal{M B}$ to have $\mathrm{R}$-charge 2 if one chooses a different $\mathrm{R}$-charge for $\Phi$ in table 2 However, $R_{\Phi} \neq 2 /(k+1)$ implies $R_{h} \neq 0$, which leads to a non-invariant superpotential since the power of $h$ in $W_{\text {conf }}$ is already fixed by $U(1)_{\Phi}$ invariance.
} 
has the correct R-charge, reduces to a superpotential of the physical degrees of freedom $M_{l}$, $B_{k}, \bar{B}_{k}$ when the constraints (3.10) are inserted and reproduces the classical constraints (3.9) through its equations of motion if (3.10) is inserted into (3.14) before the equations of motion are derived. Actually, the dependence of the superpotential on the fields is already fixed by symmetry considerations as explained in the previous section. One finds that the confining superpotential should be of the form [22] $W \sim(\bar{Q} Q)^{k N_{f}} \Phi^{(k-1) k N_{f}-k+1}$ to be consistent with the global symmetries. This agrees with the form (3.14).

The second term in (3.14) can be transformed using $\widehat{\mathcal{M}} \operatorname{cof} \mathcal{M} \equiv \operatorname{Tr}\left(\widehat{\mathcal{M}}^{\top} \operatorname{cof} \mathcal{M}\right)=$ $\operatorname{det} \mathcal{M} \operatorname{Tr}\left(\widehat{\mathcal{M}} \mathcal{M}^{-1}\right)$. Adding the appropriate powers of $\Lambda$ and $h$, one finds

$$
W_{\text {conf }}=-\sigma_{k} \frac{\overline{\mathcal{B}} \widehat{\mathcal{M}} \mathcal{B}-\operatorname{det} \mathcal{M} \operatorname{Tr}\left(\widehat{\mathcal{M}} \mathcal{M}^{-1}\right)}{h^{N_{c}} \Lambda^{k\left(2 N_{c}-N_{f}\right)}}, \quad \text { where } \sigma_{k}=(-1)^{\frac{1}{2} k(k-1) N_{f}} .
$$

This superpotential is invariant under the full global symmetry of table 2 The sign factor $\sigma_{k}$ was added for later convenience.

For the special case $k=2$, it easy to show that

$$
\operatorname{Tr}\left(\widehat{\mathcal{M}} \mathcal{M}^{-1}\right)=\operatorname{Tr}\left(M_{1} M_{2}{ }^{-1}\right), \quad \operatorname{det} \mathcal{M}=(-1)^{N_{f}}\left(\operatorname{det} M_{2}\right)^{2} \quad(\text { if } k=2) .
$$

Inserting these identities into (3.15) yields the confining superpotential in terms of $M_{1}, M_{2}$, $B_{2}, \bar{B}_{2}$ :

$$
W_{\text {conf }}=\frac{(-1)^{N_{f}+1} \bar{B}_{2} M_{2} B_{2}+\left(\operatorname{det} M_{2}\right)^{2} \operatorname{Tr}\left(M_{1} M_{2}^{-1}\right)}{h^{N_{c}} \Lambda^{2\left(2 N_{c}-N_{f}\right)}},
$$

The superpotential (3.17) agrees with the result of [12] up to the sign in front of $\bar{B}_{k} M_{k} B_{k}$. In the appendix, we determine the confining superpotential for general $k$.

Note that for each fixed value of the parameters $N_{f}, N_{c}, k$, the superpotential (3.15), (3.17) contains only one definite power of $\Lambda$. One might wonder whether the full confining superpotential is a power series in $1 / \Lambda^{2 N_{c}-N_{f}}$. The global symmetries of table 2 constrain possible additional terms to be of the form

$$
\begin{aligned}
& \frac{(\bar{Q} Q)^{k^{\prime} N_{f}} \Phi^{k^{\prime}(k-1) N_{f}+\left(k+1-2 k^{\prime}\right)}}{h^{k^{\prime} N_{f}-1} \Lambda^{k^{\prime}\left(2 N_{c}-N_{f}\right)}} \\
\sim & \frac{\left(\operatorname{det} M_{k}\right)^{k^{\prime}}\left[\operatorname{Tr}\left(M_{k-k^{\prime}+1} M_{k^{\prime}}^{-1}\right)+\cdots+\operatorname{Tr}\left(M_{2\left(k-k^{\prime}\right)+1} M_{k}^{-1}\right)\right]}{h^{k^{\prime} N_{f}-1} \Lambda^{k^{\prime}\left(2 N_{c}-N_{f}\right)}},
\end{aligned}
$$

for some positive integer $k^{\prime}$. For the expression in terms of the meson operators $M_{l}$ we assumed $k^{\prime}<k$, which is satisfied [12] as we show below. The last term in the square bracket is only present if $2 k^{\prime}>k$. Note that only this last term is smooth in the limit of vanishing field expectation values. Since the 't Hooft anomaly matchings for $Q, \bar{Q}, \Phi$ in the UV and $M_{l}, B_{k}, \bar{B}_{k}$ in the IR are satisfied at the origin of the moduli space [12, it is very 
unlikely that there are additional massless degrees of freedom at the origin of the moduli space. Thus the confining superpotential in terms of the meson and baryon operators should be smooth at vanishing field expectation values. This implies that only for $2 k^{\prime}>k$ can there be any additional terms in the confining superpotential. ${ }^{7}$ On the other hand, the equations of motion of the confining superpotential should reproduce the classical constraints (3.9) in the limit where the fields have very large expectation values, $\left\langle M_{l}\right\rangle \gg \Lambda^{1+l}$. Thus the additional terms (3.18) can only arise for $k^{\prime} \leq k$ [12]. Summarizing, we find that the integer $k^{\prime}$ in (3.18) must satisfy

$$
k<2 k^{\prime} \leq 2 k
$$

For $k=2$, this implies that $k^{\prime}=k$ and thus the effective superpotential (3.17) is exact [12]. However, additional terms in the effective superpotential are possible if $k>2$. For $k=3$, one finds that $\left(\operatorname{det} M_{3}\right)^{2} / h^{2 N_{f}-1} \Lambda^{2\left(2 N_{c}-N_{f}\right)}$ is of the form (3.18) with $k^{\prime}=2$ and thus consistent with all global symmetries. It would be interesting to see whether such a term is indeed present in the confining superpotential or whether it is excluded for a different reason.

In [12, it was shown that the confining superpotential (3.17) can be understood via the electric-magnetic duality of the Kutasov model [6, 7, 8]. The dual magnetic theory has gauge group $S U\left(\tilde{N}_{c}\right)$, with $\tilde{N}_{c}=k N_{f}-N_{c}$. This means that for $k N_{f}=N_{c}+1$, the magnetic gauge group is completely broken. The first term in (3.17) corresponds to a tree-level term of the magnetic superpotential. The second term in (3.17) is generated by a $k$-instanton effect in the completely higgsed magnetic gauge theory.

The importance of the confining superpotential for the theory with $k N_{f}=N_{c}+1$ is that the effective superpotentials for $k N_{f}<N_{c}$ can be obtained from it by successively integrating out quark flavors. For the case of SQCD (corresponding to $k=1$ ), the techniques are reviewed in [13]. For $k=2$, the authors of [12] computed the effective superpotential of the theory with $2 N_{f}=N_{c}-1$ by giving mass to one quark flavor and integrating out the heavy degrees of freedom. Generalizing their results by integrating out further quark flavors, we find that for general $N_{f}$ and $N_{c}$, the $k=2$ effective superpotential is given by

$$
\begin{aligned}
W_{\text {eff }} & =\sigma_{k}^{\frac{1}{\left(N_{c}-2 N_{f}\right)}}\left(\frac{h^{N_{c}} \Lambda^{2\left(2 N_{c}-N_{f}\right)}}{\operatorname{det} \mathcal{M}}\right)^{\frac{1}{N_{c}-2 N_{f}}} \operatorname{Tr}\left(\widehat{\mathcal{M}} \mathcal{M}^{-1}\right) \\
& =\left(\frac{h^{N_{c}} \Lambda^{2\left(2 N_{c}-N_{f}\right)}}{\left(\operatorname{det} M_{2}\right)^{2}}\right)^{\frac{1}{N_{c}-2 N_{f}}} \operatorname{Tr}\left(M_{1} M_{2}^{-1}\right) . \quad(k=2)
\end{aligned}
$$

This superpotential could only be derived for values of $N_{f}$ and $N_{c}$ such that $N_{c}+1-2 N_{f}$ is even. However, one can verify that $W_{\text {eff }}$ of (3.20) is invariant under the full global symmetry of table 2. Therefore, we conjecture that it is the exact effective superpotential for all

\footnotetext{
${ }^{7}$ We thank C. Csáki and H. Murayama for a useful discussion on this point.
} 
$2 N_{f}<N_{c}$. This generalizes the effective superpotential for $S Q C D$ with $N_{f}<N_{c}$ found by Affleck, Dine and Seiberg [1] to the Kutasov model. For $2 N_{f}=N_{c}-1$ the superpotential (3.20) has the form of a 2-instanton term.

For $2 N_{f}<N_{c}-1$, one might speculate whether the effective superpotential is generated by gluino condensation in the unbroken $S U\left(N_{c}-2 N_{f}\right)$ gauge theory at a generic point of the moduli space. This would be very similar to the situation of SQCD with $N_{f}<N_{c}-1$. In SQCD, gluino condensation in the unbroken $S U\left(N_{c}-N_{f}\right)$ gauge theory generates a superpotential $W=\Lambda_{L}^{3}$, which via the scale matching relation $\Lambda^{3 N_{c}-N_{f}}=\Lambda_{L}^{3\left(N_{c}-N_{f}\right)} \operatorname{det} M$, leads to the Affleck-Dine-Seiberg superpotential $W=\left(\Lambda^{3 N_{c}-N_{f}} / \operatorname{det} M\right)^{1 /\left(N_{c}-N_{f}\right)}$. If the same mechanism is at work in the $k=2$ Kutasov model, we need a much more complicated scale matching relation. If the high-energy $S U\left(N_{c}\right)$ gauge theory with dynamical scale $\Lambda$ is broken to an $S U\left(N_{c}-2 N_{f}\right)$ gauge theory with scale $\Lambda_{L}$ by generic expectation values for $M_{1}$ and $M_{2}$, then the two scales should be related by

$$
\Lambda^{2\left(2 N_{c}-N_{f}\right)}=\Lambda_{L}^{3\left(N_{c}-2 N_{f}\right)} h^{-N_{c}}\left(\operatorname{det} M_{2}\right)^{2}\left(\operatorname{Tr}\left(M_{1} M_{2}^{-1}\right)\right)^{2 N_{f}-N_{c}}
$$

if we want that the effective superpotential (3.20) is generated by the gluino condensation term $W=\Lambda_{L}^{3}$. Assuming that the gauge couplings $g$ of the $S U\left(N_{c}\right)$ theory and $g_{L}$ of the $S U\left(N_{c}-2 N_{f}\right)$ theory are matched by the relation ${ }^{8} 1 / g^{2}(v)=1 /\left(2 g_{L}^{2}(v)\right)$, where $v$ is the symmetry breaking scale, one has [23]

$$
\left(\frac{\Lambda^{2 N_{c}-N_{f}}}{v^{2 N_{c}-N_{f}}}\right)^{2}=\frac{\Lambda_{L}^{3\left(N_{c}-2 N_{f}\right)}}{v^{3\left(N_{c}-2 N_{f}\right)}}
$$

This implies that the symmetry breaking scale $v$ is related to the meson expectation values via

$$
\left(\operatorname{det}\left(\frac{M_{2}}{h v^{3}}\right)\right)^{2}=\left(h v \operatorname{Tr}\left(M_{1} M_{2}^{-1}\right)\right)^{N_{c}-2 N_{f}}
$$

While this does not seem unreasonable, we do not have any independent argument to justify this relation.

Let us briefly consider the cases $2 N_{f} \geq N_{c}$. The authors of [24] have shown that for $2 N_{f}=N_{c}$, the $k=2$ Kutasov model has a quantum modified moduli space with vanishing effective superpotential. For $2 N_{f}>N_{c}$, the superpotential (3.20) is still consistent with all symmetries. But it does not take into account the baryon operators. For $2 N_{f}=N_{c}+1$, it is just the second term in the confining superpotential (3.17). Assuming that (3.17) is still the correct superpotential for $2 N_{f}>N_{c}$ in the limit where the vacuum expectation values of all baryon operators vanish, we can obtain some non-trivial information about the theories (2.1) with $m_{\Phi}=0$ by using electric-magnetic duality. This will be shown in the next section.

${ }^{8}$ The factor 2 in the matching of the gauge couplings $g$ and $g_{L}$ is related to the fact that the effective superpotential is generated by a 2 -instanton effect in the case $2 N_{f}=N_{c}-1$. 


\begin{tabular}{c|cccccc} 
& $S U\left(N_{f}\right)_{L}$ & $S U\left(N_{f}\right)_{R}$ & $U(1)_{R}$ & $U(1)_{\Phi}$ & $U(1)_{A}$ & $U(1)_{B}$ \\
\hline$q$ & $\square$ & $\mathbf{1}$ & $1-\frac{2 \tilde{N}_{c}}{(k+1) N_{f}}$ & 0 & -1 & $\frac{1}{\tilde{N}_{c}}$ \\
$\bar{q}$ & $\mathbf{1}$ & $\square$ & $1-\frac{2 \tilde{N}_{c}}{(k+1) N_{f}}$ & 0 & -1 & $-\frac{1}{\tilde{N}_{c}}$ \\
$\tilde{\Phi}$ & $\mathbf{1}$ & $\mathbf{1}$ & $\frac{2}{k+1}$ & 1 & 0 & 0 \\
$\tilde{h}$ & $\mathbf{1}$ & $\mathbf{1}$ & 0 & $-(k+1)$ & 0 & 0 \\
$\tilde{M}_{l}$ & $\square$ & $\square$ & $\left(-\frac{2 N_{c}}{N_{f}}+k+l\right) \frac{2}{k+1}$ & $2(l-k)$ & 2 & 0 \\
$\mu$ & $\mathbf{1}$ & $\mathbf{1}$ & 0 & -1 & 0 & 0 \\
$\tilde{\Lambda}^{b}$ & $\mathbf{1}$ & $\mathbf{1}$ & 0 & $2 \tilde{N}_{c}$ & $2 N_{f}$ & 0
\end{tabular}

Table 3: Charges of the fields and parameters of the dual magnetic theory (4.1).

\section{Effective superpotentials from duality}

The $S U\left(N_{c}\right)$ gauge theory with one adjoint field $\Phi, N_{f}$ quark flavors $Q, \bar{Q}$ and tree-level superpotential $W_{\text {tree }}=\frac{h}{k+1} \operatorname{tr} \Phi^{k+1}$ discussed in section 3 has a dual description in terms of magnetic variables [6, 7, 8. The dual magnetic theory has gauge group $\operatorname{SU}\left(\tilde{N}_{c}\right)$, with $\tilde{N}_{c}=k N_{f}-N_{c}$, one adjoint field $\tilde{\Phi}, N_{f}$ quark flavors $q, \bar{q}, k N_{f}^{2}$ gauge singlets $\tilde{M}_{l}$ with charges as shown in table 3 and tree-level superpotential

$$
W_{\mathrm{mag}}=\frac{1}{k+1} \tilde{h} \operatorname{tr} \tilde{\Phi}^{k+1}+\sum_{l=1}^{k} \mu^{l-k} \tilde{M}_{l} \bar{q} \tilde{\Phi}^{k-l} q .
$$

The scale $\mu$ had to be introduced to obtain the correct mass dimensions. It will be related to the dynamical scales $\Lambda$ of the electric theory and $\tilde{\Lambda}$ of the magnetic theory via the duality map. Note that the gauge singlets $\tilde{M}_{l}$ have the canonical mass dimension of a chiral superfield although they correspond to the generalized mesons $M_{l}$ of the electric theory, which have mass dimensions $1+l$. The precise mapping is given below.

The generalized magnetic mesons $\bar{q} \tilde{\Phi}^{l-1} q$ are not moduli of the magnetic theory; their expectation values do not correspond to flat directions but rather are fixed by the superpotential (4.1). However, there are magnetic moduli corresponding to vacuum expectation values of baryon operators. Let us introduce dressed quark operators as we did in the electric theory in eq. (3.3):

$$
q_{l} \equiv \tilde{\Phi}^{l-1} q, \quad \bar{q}_{l} \equiv \bar{q} \tilde{\Phi}^{l-1} .
$$


Now, the magnetic baryons are given by

$$
\begin{aligned}
& b^{\left(m_{1}, \ldots, m_{k}\right)} \equiv\left(q_{1}\right)^{m_{1}}\left(q_{2}\right)^{m_{2}} \cdots\left(q_{k}\right)^{m_{k}}, \quad \bar{b}^{\left(\bar{m}_{1}, \ldots, \bar{m}_{k}\right)} \equiv\left(\bar{q}_{1}\right)^{\bar{m}_{1}}\left(\bar{q}_{2}\right)^{\bar{m}_{2} \cdots\left(\bar{q}_{k}\right)^{\bar{m}_{k}},} \\
& \text { with } \sum_{l=1}^{k} m_{k}=\sum_{l=1}^{k} \bar{m}_{k}=\tilde{N}_{c} .
\end{aligned}
$$

The integers $\left\{m_{l}\right\}$ label the different types of baryons. Each of them carries $k N_{f}-\tilde{N}_{c}$ flavor indices which we have not displayed. The exponents denote the powers to which the dressed quarks appear. The color indices are contracted with a rank $\tilde{N}_{c}$ epsilon-tensor. The $\tilde{N}_{c}$ flavor indices are contracted with $k$ rank $N_{f}$ epsilon-tensors, leaving $k N_{f}-\tilde{N}_{c}$ free indices.

The precise mapping between the electric and the magnetic theory is [8]

$$
\begin{aligned}
\tilde{N}_{c}= & k N_{f}-N_{c}, \\
\Lambda^{2 N_{c}-N_{f}} \tilde{\Lambda}^{2 \tilde{N}_{c}-N_{f}}= & h^{-2 N_{f}} \mu^{2 N_{f}}, \\
\tilde{h}= & -h, \\
\tilde{M}_{l}= & h \mu^{k-l-2} M_{l}, \\
b^{\left(m_{1}, \ldots, m_{k}\right)}= & \left((-1)^{\frac{1}{2} k(k-1) N_{f}-N_{c}} \tilde{h}^{k N_{f}} \mu^{-2 \tilde{N}_{c}} \Lambda^{k\left(2 N_{c}-N_{f}\right)}\right)^{-1 / 2} B^{\left(n_{1}, \ldots, n_{k}\right)}, \\
& \operatorname{with} m_{l}=N_{f}-n_{k+1-l}, \\
\operatorname{tr} \tilde{\Phi}^{l}= & -\operatorname{tr} \Phi^{l}, \quad l=2, \ldots, k-1, \quad \operatorname{tr} \tilde{\Phi}^{k}=\frac{\tilde{N}_{c}}{N_{c}} \operatorname{tr} \Phi^{k} .
\end{aligned}
$$

This mapping respects all the global symmetries of tables 2 and 3. Again we suppressed the flavor indices of the baryons. In the right hand side of the fifth line of (4.4), $B^{\left(n_{1}, \ldots, n_{k}\right)}$ is contracted with $k$ epsilon-tensors of ranks $N_{f}-n_{l}+m_{k-l+1}, l=1, \ldots, k$. Let us verify that both sides of the baryon mapping have the same mass dimension. From the definitions of $B^{\left(n_{1}, \ldots, n_{k}\right)}$ and $b^{\left(m_{1}, \ldots, m_{k}\right)}$ in eqs. (3.4), (4.3), one finds

$$
\begin{aligned}
\operatorname{dim}\left(B^{\left(n_{1}, \ldots, n_{k}\right)}\right) & =\sum_{l=1}^{k} l n_{l}, \\
\operatorname{dim}\left(b^{\left(m_{1}, \ldots, m_{k}\right)}\right) & =\sum_{l=1}^{k} l m_{l}=\sum_{l=1}^{k}\left(l N_{f}-(k+1-l) n_{l}\right) \\
& =\frac{1}{2}(k+1)\left(\tilde{N}_{c}-N_{c}\right)+\operatorname{dim}\left(B^{\left(n_{1}, \ldots, n_{k}\right)}\right), \\
\operatorname{dim}\left(\tilde{h}^{k N_{f}} \mu^{-2 \tilde{N}_{c}} \Lambda^{k\left(2 N_{c}-N_{f}\right)}\right) & =k N_{f}(2-k)-2\left(k N_{f}-N_{c}\right)+k\left(2 N_{c}-N_{f}\right) \\
& =(k+1)\left(N_{c}-\tilde{N}_{c}\right) .
\end{aligned}
$$

Similarly, one can show that $(\ldots)^{-1 / 2} B^{\left(n_{1}, \ldots, n_{k}\right)}$ and $b^{\left(m_{1}, \ldots, m_{k}\right)}$ in eq. (4.4) have the same charges under all global symmetries. It is also instructive to verify that under two successive 
duality transformations the electric baryons are mapped onto themselves. Performing the duality mapping (4.4) twice, one finds $B^{\left(n_{1}, \ldots, n_{k}\right)} \rightarrow(-1)^{-\frac{1}{2} k(k-1) N_{f}} B^{\left(n_{1}, \ldots, n_{k}\right)}$. The sign ambiguity is related to the possibility of field redefinitions using the global symmetry $U(1)_{B}$, as explained in [8].

The goal in this section is to use electric-magnetic duality in the Kutasov model to find some nonperturbative effective superpotential which is difficult to obtain by other methods. Using the duality mapping (4.4), we can translate the effective superpotential (3.20) of the electric theory to the magnetic theory. Written in terms of magnetic variables, the electric superpotential (3.20) reads

$$
W_{\text {eff }}=\mu^{-1}\left(h^{\tilde{N}_{c}} \tilde{\Lambda}^{2\left(2 \tilde{N}_{c}-N_{f}\right)}\left(\operatorname{det} \tilde{M}_{2}\right)^{2}\right)^{\frac{1}{N_{c}}} \operatorname{Tr}\left(\tilde{M}_{1} \tilde{M}_{2}^{-1}\right) .
$$

Such an effective superpotential should be generated by nonperturbative effects in the magnetic gauge theory. The authors of [12] showed that if the magnetic gauge group is broken down to $\tilde{N}_{c}=1$ by the Higgs effect, then the superpotential (4.6) is indeed generated by a 2-instanton effect. Interestingly, the only term of the magnetic tree-level superpotential (4.1) that remains in the completely higgsed theory with $\tilde{N}_{c}=1$, namely $\tilde{M}_{2} \bar{q} q$, maps precisely to the term proportional to $B_{2} M_{2} B_{2}$ in (3.17). From (4.3), we see that the magnetic baryons $b^{(1,0)}, \bar{b}^{(1,0)}$ are just given by the quark singlets $q, \bar{q}$ that remain light after the Higgs effect. Then, using (4.4), we find

$$
\tilde{M}_{2} \bar{q} q=\frac{(-1)^{N_{f}+1} \bar{B}_{2} M_{2} B_{2}}{h^{N_{c}} \Lambda^{2\left(2 N_{c}-N_{f}\right)}}
$$

which is exactly the first term in (3.17). Summarizing, the first term in the confining superpotential (3.17) of the electric theory corresponds to the tree-level term $\tilde{M}_{2} \bar{q} q$ in the magnetic theory and the second term in (3.17) corresponds to the 2-instanton term (4.6) in the magnetic theory (with $\tilde{N}_{c}=1$ ).

In general (for $\tilde{N}_{c}>1$ ), the effective superpotential (4.6) also contains contributions including baryon operators. We have not determined these contributions. However, (4.6) is correct in the limit where the vacuum expectation values of all baryon operators vanish.

Comparing the magnetic singlets $\tilde{M}_{l}$ in (4.1) with the coupling parameters $m_{l}$ in (2.1), we see that for $m_{l}=\mu^{1-l} \tilde{M}_{k+1-l}$ and $m_{\Phi}=0$ the two superpotentials agree if we replace $q, \bar{q}, \tilde{\Phi}$ by $Q, \bar{Q}, \Phi$ (not using the duality map but just renaming the elementary fields). Thus, (4.6) gives the effective superpotential for the case of general $m_{l}$ in (2.1) but massless adjoint field.$^{9}$ Explicitly, one finds that the effective superpotential for the theory (2.1) with

\footnotetext{
${ }^{9}$ We implicitly assumed that the $m_{l}$ are background fields. The low-energy result for constant $m_{l}$ is obtained by taking these background fields to be very heavy and effectively replacing them by their expectation values.
} 
$m_{\Phi}=0$ and $k=2$ is given by

$$
\begin{aligned}
W_{\mathrm{eff}} & =\left(h^{N_{c}} \Lambda^{2\left(2 N_{c}-N_{f}\right)}\left(\operatorname{det} m_{1}\right)^{2}\right)^{\frac{1}{N_{c}}} \operatorname{Tr}\left(m_{2} m_{1}^{-1}\right) \\
& =h \Lambda_{L}^{4} \operatorname{Tr}\left(m_{2} m_{1}^{-1}\right)
\end{aligned}
$$

where we dropped the tildes over $N_{c}$ and $\Lambda$ and we used the scale matching condition

$\Lambda_{L}^{2 N_{c}}=\Lambda^{2 N_{c}-N_{f}} \operatorname{det} m_{1}$. When $m_{1}, m_{2}$ are considered to be constants, the right hand side of (4.8) represents only the leading term in an expansion in $\Lambda_{L}$. Higher powers of $\Lambda_{L}$ are generated upon integrating out the quantum fluctuations of the background fields $m_{l}$. Also, the electric-magnetic duality used to arrive at the result (4.8) is only valid close to the IR fixed point, which again implies that it is only the leading term. The important result of this analysis is that the leading term in the effective superpotential (4.8) is proportional to $\Lambda_{L}^{4}$. The 1-instanton term proportional to $\Lambda_{L}^{2}$, which was expected by naive dimensional analysis is not present. This result can be verified by factorizing the corresponding Seiberg-Witten curve of this model [20].

\section{Conclusions and discussion}

In this paper, we derived the effective superpotential for an $\mathcal{N}=1 S U\left(N_{c}\right)$ gauge theory with one massless adjoint matter field and $N_{f}$ massless fundamental flavors and a cubic superpotential for the adjoint field. This generalizes the well-known effective superpotential for SQCD with $N_{f}<N_{c}$ found by Affleck, Dine and Seiberg to the model first discussed by Kutasov. The global symmetries alone are not enough to fix the precise form of the superpotential of the Kutasov model. However the additional requirement that the equations of motion reproduce the classical constraints on the moduli space in the special case $2 N_{f}=$ $N_{c}+1$ uniquely determines the effective superpotential. If the tree-level superpotential for the adjoint field is quartic or of higher order, the global symmetries and the constraints on the moduli space are not sufficient to determine the effective superpotential. However, one can obtain the leading term in an expansion in powers of $\Lambda$, as we have shown in the appendix. Moreover, we were able to determine the exact constraints on the quantum moduli space for $k N_{f}=N_{c}+1$ and we showed that they coincide with the classical constraints.

Using Kutasov duality, the effective superpotential for the theory with massless adjoint and fundamental fields and cubic superpotential can be written in terms of magnetic variables. The magnetic theory has almost the same matter content but the rank of the gauge group is different and there are additional gauge singlets, which are elementary fields. These gauge singlets couple to the quarks and to the adjoint field via Yukawa-like couplings and effectively provide masses for the quarks. Thus, via Kutasov duality, we found the leading 
term of the effective superpotential of a supersymmetric gauge theory with one massless adjoint field and $N_{f}$ massive quark flavors and a superpotential which couples the quarks to the adjoint field.

Recently, Dijkgraaf and Vafa discovered a surprising link between effective superpotentials of supersymmetric gauge theories and the effective potential of an associated bosonic matrix model [16. This is very interesting since it allows one to obtain nonperturbative results by doing a perturbative calculation. It is a powerful method to compute the effective superpotential of $\mathcal{N}=1$ gauge theories with massive matter in tensor and fundamental representations. However, it is difficult to use this method if the gauge theory contains massless fields. ${ }^{10}$ More recently, it is understood why such a simplification can arise as a consequence of (super)symmetries by considering Ward identities associated to the Konishi anomaly [26] (see also [27), which turns out to be the same as the loop equation in the matrix model. The loop equation is identical to the minimization of the superpotential, which in turn requires that all periods of the generating 1-form $T$ on the Riemann surface defined by the superpotential are integers. By Abel's theorem, the 1-form must be a derivative of a meromorphic function $\psi$. Finally, the condition that $\psi$ be single valued on the reduced Riemann surface is the factorization of the Seiberg-Witten curve of the original $\mathcal{N}=2$ theory to the reduced curve defined by the superpotential. This completes the solution to the problem and corresponds to extending the earlier results of Vafa and his collaborators [18, 19.

If we can show this effective superpotential agrees with the result from an explicit factorization of the corresponding Seiberg-Witten curve, one can provide further evidence for the validity of the electric-magnetic duality discovered by Kutasov. This program is under progress and will be published in separate paper [20].

\section{Acknowledgements}

We would like to thank C. Csáki, H. Murayama and R. Tatar for helpful discussions. The work of MK is funded by the Deutsche Forschungsgemeinschaft. The work of SJS is supported by by KOSEF Grant 1999-2-112-003-5.

\footnotetext{
${ }^{10}$ See however [25], where the case of massless fundamental fields is discussed.
} 


\section{A Effective superpotentials for $k>2$}

In this appendix, we would like to generalize some of the formulae that were only derived for the $k=2$ Kutasov model to the situation where $W_{\text {tree }}=\frac{h}{k+1} \operatorname{tr} \Phi^{k+1}$, for general $k$.

Generalizing, eq. (3.16), one finds

$$
\begin{aligned}
& \operatorname{Tr}\left(\widehat{\mathcal{M}} \mathcal{M}^{-1}\right)=(k-1) \operatorname{Tr}\left(M_{1} M_{k}^{-1}\right)-(k-2) \operatorname{Tr}\left(M_{2} M_{k}{ }^{-1} M_{k-1} M_{k}{ }^{-1}\right) \\
& +(k-3)\left[\operatorname{Tr}\left(M_{3} M_{k}^{-1} M_{k-1} M_{k}^{-1} M_{k-1} M_{k}^{-1}\right)-\operatorname{Tr}\left(M_{3} M_{k}^{-1} M_{k-2} M_{k}{ }^{-1}\right)\right] \\
& -(k-4)\left[\operatorname{Tr}\left(M_{4} M_{k}^{-1} M_{k-1} M_{k}^{-1} M_{k-1} M_{k}^{-1} M_{k-1} M_{k}^{-1}\right)\right. \\
& -\operatorname{Tr}\left(M_{4} M_{k}^{-1} M_{k-2} M_{k}^{-1} M_{k-1} M_{k}^{-1}\right) \\
& -\operatorname{Tr}\left(M_{4} M_{k}^{-1} M_{k-1} M_{k}^{-1} M_{k-2} M_{k}^{-1}\right) \\
& \left.+\operatorname{Tr}\left(M_{4} M_{k}^{-1} M_{k-3} M_{k}^{-1}\right)\right] \\
& +(k-5)[\cdots]+-\cdots+(-1)^{k}[\cdots] \text {. }
\end{aligned}
$$

The determinant of the meson matrix in (3.10) is now given by

$$
\operatorname{det} \mathcal{M}=(-1)^{\frac{1}{2} k(k-1) N_{f}}\left(\operatorname{det} M_{k}\right)^{k} \text {. }
$$

Inserting these results into (3.15), we find

$$
W_{\text {conf }}=\frac{-(-1)^{\frac{1}{2} k(k-1) N_{f}} \bar{B}_{k} M_{k} B_{k}+\left(\operatorname{det} M_{k}\right)^{k}\left[(k-1) \operatorname{Tr}\left(M_{1} M_{k}^{-1}\right)+\ldots\right]}{h^{N_{c}} \Lambda^{k\left(2 N_{c}-N_{f}\right)}},
$$

where the '...' represent the terms proportional to $(k-2),(k-3), \ldots$, in (A.1). The superpotential (A.3) agrees with the result of [12] up to the sign in front of $\bar{B}_{k} M_{k} B_{k}$. The method described above provides us with a simple algorithm to compute the explicit coefficients of the various terms for the cases with $k>2$.

Successively integrating out quark flavors from (A.3), we obtain an effective superpotential for general $k, N_{f}$ and $N_{c}$ :

$$
\begin{aligned}
W_{\text {eff }} & =(-1)^{\frac{k(k-1) N_{f}}{2\left(N_{c}-k N_{f}\right)}}\left(\frac{h^{N_{c}} \Lambda^{k\left(2 N_{c}-N_{f}\right)}}{\operatorname{det} \mathcal{M}}\right)^{\frac{1}{N_{c}-k N_{f}}} \operatorname{Tr}\left(\widehat{\mathcal{M}} \mathcal{M}^{-1}\right) \\
& =\left(\frac{h^{N_{c}} \Lambda^{k\left(2 N_{c}-N_{f}\right)}}{\left(\operatorname{det} M_{k}\right)^{k}}\right)^{\frac{1}{N_{c}-k N_{f}}}\left[(k-1) \operatorname{Tr}\left(M_{1} M_{k}^{-1}\right)+\ldots\right] .
\end{aligned}
$$

However, as explained in section 3, for $k>2$, the confining superpotential (A.3) contains additional terms of the form (3.18). Thus, the effective superpotential (A.4) is not the full answer but only contains the terms with the highest power in $\Lambda$. 
As a consequence of the additional terms (3.18) in the confining superpotential (A.3), the classical constraints (3.9) might be modified quantum mechanically. This would be in sharp contrast to the case of SQCD and to the $k=2$ Kutasov model, where the classical constraints remain exact in the full quantum theory. However, a more detailed examination shows that the classical constraints do not receive any quantum corrections. To see this, let us first consider the confining superpotential for $k=3$ and $3 N_{f}=N_{c}+1$. It consists of of a term proportional to $\Lambda^{-3\left(2 N_{c}-N_{f}\right)}$ as given in (A.3) and one additional term proportional to $\Lambda^{-2\left(2 N_{c}-N_{f}\right)}$ as given in (3.18), with $k^{\prime}=2$.

$$
\begin{aligned}
W_{\text {conf }}= & \frac{(-1)^{N_{f}+1} \bar{B}_{3} M_{3} B_{3}+\left(\operatorname{det} M_{3}\right)^{3}\left[2 \operatorname{Tr}\left(M_{1} M_{3}{ }^{-1}\right)-\operatorname{Tr}\left(M_{2} M_{3}{ }^{-1} M_{2} M_{3}{ }^{-1}\right)\right]}{h^{N_{c}} \Lambda^{3\left(2 N_{c}-N_{f}\right)}} \\
& +a \frac{\left(\operatorname{det} M_{3}\right)^{2}}{h^{2 N_{f}-1} \Lambda^{2\left(2 N_{c}-N_{f}\right)}},
\end{aligned}
$$

where $a$ is some unknown relative coefficient. The constraints on the quantum moduli space are obtained from the equations of motion of (A.5). From $\partial W_{\text {conf }} / \partial M_{1}=0$, one finds that $\operatorname{det} M_{3}=0$ on the quantum moduli space. Thus, the quantum constraints are given by

$$
\bar{B}_{3} M_{3}=0=M_{3} B_{3}, \quad(-1)^{N_{f}+1} \bar{B}_{3} B_{3}=\left(\operatorname{cof} M_{3}\right) \operatorname{Tr}\left(M_{2}\left(\operatorname{cof} M_{3}\right) M_{2}\left(\operatorname{cof} M_{3}\right)\right) .
$$

This coincides with the classical constraints. The situation in the case of general $k$ is very similar. The additional terms (3.18) do not contain a term proportional to $M_{1}$. And from (A.1), one finds that the only term that contains $M_{1}$ in (A.3) is the one proportional to $\left(\operatorname{det} M_{k}\right)^{k} \operatorname{Tr}\left(M_{1} M_{k}^{-1}\right)$. Thus, the equation of motion for $M_{1}$ implies that $\operatorname{det} M_{k}=0$ on the quantum moduli space. This, in turn, leads to the following quantum constraints:

$$
\bar{B}_{k} M_{k}=0=M_{k} B_{k}, \quad(-1)^{\frac{1}{2} k(k-1) N_{f}+k} \bar{B}_{k} B_{k}=\left(\operatorname{cof} M_{k}\right) \operatorname{Tr}\left(\left(M_{k-1} \operatorname{cof} M_{k}\right)^{k-1}\right),
$$

which again coincides with the classical constraints.

The effective superpotential (A.4) can be written in terms of magnetic variables using the duality mapping (4.4).

$$
W_{\text {eff }}=\mu^{1-k}\left(h^{\tilde{N}_{c}} \tilde{\Lambda}^{k\left(2 \tilde{N}_{c}-N_{f}\right)}\left(\operatorname{det} \tilde{M}_{k}\right)^{k}\right)^{\frac{1}{N_{c}}}\left[(k-1) \operatorname{Tr}\left(\tilde{M}_{1} \tilde{M}_{k}^{-1}\right)+\ldots\right] .
$$

Again we can replace the magnetic singlets by their expectation values $m_{l}=\mu^{1-l} \tilde{M}_{k+1-l}$ as we did at the end of section 4. If we also replace $q, \bar{q}, \tilde{\Phi}$ by $Q, \bar{Q}, \Phi$ (not using the duality map but just renaming the elementary fields), we obtain

$$
\begin{aligned}
W_{\text {eff }} & =\left(h^{N_{c}} \Lambda^{k\left(2 N_{c}-N_{f}\right)}\left(\operatorname{det} m_{1}\right)^{k}\right)^{\frac{1}{N_{c}}}\left[(k-1) \operatorname{Tr}\left(m_{k} m_{1}^{-1}\right)+\ldots\right] \\
& =h \Lambda_{L}^{2 k}\left[(k-1) \operatorname{Tr}\left(m_{k} m_{1}^{-1}\right)+\ldots\right] .
\end{aligned}
$$

However this result is not as useful as the corresponding one for the $k=2$ case since we neglected the additional terms (3.18) in (A.3) which lead to terms with lower powers of $\Lambda$ in (A.4) and thus to additional terms with lower powers of $\Lambda_{L}$ in (A.9). 


\section{References}

[1] I. Affleck, M. Dine, N. Seiberg, "Dynamical Supersymmetry Breaking In Supersymmetric QCD", Nucl.Phys. B241 (1984) 493; "Dynamical Supersymmetry Breaking In FourDimensions And Its Phenomenological Implications", Nucl.Phys. B256 (1985) 557.

[2] N. Seiberg, "Naturalness Versus Supersymmetric Non-renormalization Theorems", Phys.Lett. B318 (1993) 469, hep-ph/9309335.

[3] N. Seiberg, "Exact Results on the Space of Vacua of Four Dimensional SUSY Gauge Theories", Phys.Rev. D49 (1994) 685, hep-th/9402044.

[4] G. 't Hooft, in "Recent Developments in Gauge Theories", Proc. of Cargèse Summer School 1979, edited by G. 't Hooft et al., Plenum Press, New York (1980).

[5] N. Seiberg, "Electric-Magnetic Duality in Supersymmetric Non-Abelian Gauge Theories", Nucl.Phys. B435 (1995) 129, hep-th/9411149.

[6] D. Kutasov, "A Comment on Duality in $N=1$ Supersymmetric Non-Abelian Gauge Theories", Phys.Lett. B351 (1995) 230, hep-th/9503086.

[7] D. Kutasov, A. Schwimmer, "On Duality in Supersymmetric Yang-Mills Theory", Phys.Lett. B354 (1995) 315, hep-th/9503086.

[8] D. Kutasov, A. Schwimmer, N. Seiberg, "Chiral Rings, Singularity Theory and ElectricMagnetic Duality", Nucl.Phys. B459 (1996) 455, hep-th/9510222.

[9] S. Elitzur, A. Forge, A. Giveon, E. Rabinovici, "Effective Potentials and Vacuum Structure in $N=1$ Supersymmetric Gauge Theories", Nucl.Phys. B459 (1996) 160, hep-th/9509130.

[10] Y. Demasure, R. A. Janik, "Explicit factorization of Seiberg-Witten curves with matter from random matrix models", hep-th/0212212.

[11] F. Cachazo, N. Seiberg, E. Witten, "Chiral Rings and Phases of Supersymmetric Gauge Theories", JHEP 0304 (2003) 018, hep-th/0303207.

[12] C. Csáki, H. Murayama, "New Confining N=1 Supersymmetric Gauge Theories", Phys.Rev. D59 (1999) 065001, hep-th/9810014.

[13] N. Seiberg, K. Intriligator, "Lectures on supersymmetric gauge theories and electricmagnetic duality", Nucl.Phys.Proc.Suppl. 45BC (1996) 1, hep-th/9509066. 
[14] I. Bena, R. Roiban, "Exact superpotentials in $N=1$ theories with flavor and their matrix model formulation", Phys.Lett. B555 (2003), hep-th/0211075; I. Bena, R. Roiban, R. Tatar, "Baryons, Boundaries and Matrix Models", hep-th/0211271.

[15] G. Veneziano, S. Yankielowicz, "An Effective Lagrangian For The Pure $N=1$ Supersymmetric Yang-Mills Theory", Phys.Lett. B113 (1982) 231.

[16] R. Dijkgraaf, C. Vafa, "A Perturbative Window into Non-Perturbative Physics", hep-th/0208048.

[17] R. Argurio, V. L. Campos, G. Ferretti, R. Heise, "Exact Superpotentials for Theories with Flavors via a Matrix Integral", Phys.Rev. D67 (2003) 065005, hep-th/0210291.

[18] F. Cachazo, K. A. Intriligator and C. Vafa, "A large N duality via a geometric transition" Nucl.Phys. B603 (2001) 3, hep-th/0103067.

[19] F. Cachazo and C. Vafa, " $N=1$ and $N=2$ geometry from fluxes", hep-th/0206017.

[20] M. Klein, S.-J. Sin, in preparation.

[21] K. Intriligator, "Integrating in' and exact superpotentials in 4d”, Phys.Lett. B336 (1994) 409, hep-th/9407106.

[22] M. Klein, "More Confining N=1 SUSY Gauge Theories From Non-Abelian Duality", Nucl.Phys. B553 (1999) 155, hep-th/9812155.

[23] C. Csáki, H. Murayama, "Instantons in Partially Broken Gauge Groups, Nucl.Phys. B532 (1998) 498, hep-th/9804061

[24] H. Murayama, E. Perazzi, "New Theories With Quantum Modified Moduli Space", JHEP 0205 (2002) 055, hep-th/0112082.

[25] B. Feng, "Seiberg Duality in Matrix Model", hep-th/0211202; B. Feng, Y.-H. He, "Seiberg Duality in Matrix Models II", hep-th/0211234; B. Feng, "Note on Matrix Model with Massless Flavors", hep-th/0212274; "Note on Seiberg Duality in Matrix Model", hep-th/0303144.

[26] F. Cachazo, M. R. Douglas, N. Seiberg, E. Witten, "Chiral Rings and Anomalies in Supersymmetric Gauge Theory", JHEP 0212 (2002) 071, hep-th/0211170; F. Cachazo, N. Seiberg, E. Witten, "Phases of N=1 Supersymmetric Gauge Theories and Matrices", JHEP 0302 (2003) 042, hep-th/0301006.

[27] R. Dijkgraaf, M. T. Grisaru, C. S. Lam, C. Vafa and D. Zanon, "Perturbative computation of glueball superpotentials", hep-th/0211017. 